\title{
Guided Wave based Damage Detection in a Composite T-joint using 3D Scanning Laser Doppler Vibrometer
}

\author{
G. Kolappan Geetha, D. Roy Mahapatra*, S. Gopalakrishnan \\ Department of Aerospace Engineering, Indian Institute of Science, Bangalore 560 012, India
}

\begin{abstract}
Composite T-joints are commonly used in modern composite airframe, pressure vessels and piping structures, mainly to increase the bending strength of the joint and prevents buckling of plates and shells, and in multi-cell thin-walled structures. Here we report a detailed study on the propagation of guided ultrasonic wave modes in a composite T-joint and their interactions with delamination in the co-cured co-bonded flange. A well designed guiding path is employed wherein the waves undergo a two step mode conversion process, one is due to the web and joint filler on the back face of the flange and the other is due to the delamination edges close to underneath the accessible surface of the flange. A 3D Laser Doppler Vibrometer is used to obtain the three components of surface displacements/velocities of the accessible face of the flange of the T-joint. The waves are launched by a piezo ceramic wafer bonded on to the back surface of the flange. What is novel in the proposed method is that the location of any change in material/geometric properties can be traced by computing a frequency domain power flow along a scan line. The scan line can be chosen over a grid either during scan or during post-processing of the scan data off-line. The proposed technique eliminates the necessity of baseline data and disassembly of structure for structural interrogation.
\end{abstract}

Keywords: Guided wave, T-joint, delamination, composite, LDV, power flow, mode conversion, wavenumber.

\section{INTRODUCTION}

With ever-expanding aviation industry, the day-to-day usage of aircraft is increasing. This necessitates a potential market with aircraft of better structural materials and improvised structural design. In the improvised version of the structural design, the metallic alloy structural components are replaced by composites materials which are having high strength to weight ratio and better structural properties. In addition to structural weight saving it also possess better corrosion and fire resistant properties and provides better flight maneuverability. The assembly of composite structures is not a straight forward task. Like metallic structures it is not easy and feasible to join different composite components using rivet joints or different cross-sectional angle plates. The assembly of complex composite structure dictates the need for efficient joining methods like T-joints and T-stiffeners. The incorporation of co-cured, co-bonded complex composite components in aviation vehicles have resulted in the total reduction of structural components by $40 \%$ compared to metallic structure. The use of efficient joining techniques have reduced the usage of mechanical fasteners and drilled holes by $50 \%$. The type of composite $\mathrm{T}$-joint considered in the present work is common to modern composite airframe, pressure vessels and piping structures. The purpose of the T-joint is to prevent skin buckling during wing loading and to increases the bending strength of the joint. T-joint consists of a flange, web, and radius filler. The flange interfaces with the skin, the web provides an interface for attachment to the substructure, and the radius filler provides continuity of load transfer between the web and flange. The T-joint enhances the transfer of flexural, tension, and shear loads to the skin.

Maintenance of aircraft structure accounts for about one-third of aircraft cost. Recent studies by the aircraft industry [1] have demonstrated that Structural Health Monitoring (SHM) systems could result in considerable maintenance cost

\footnotetext{
${ }^{*}$ Corresponding Author E-mail: droymahapatra@aero.iisc.ernet.in
} 
reduction. As per the survey about $90 \%$ of skin inspections are currently carried using visual inspection and remaining $10 \%$ using non-destructive inspection techniques like eddy current, dye penetration, radiography, ultrasonic etc [2]. Scanning an entire structural panel using techniques like pulse-echo and pitch-catch methods is time consuming and it has a limitation that the inspection of damages in the hidden parts or sub-surfaces is not straightforward. At least an elaborate through-thickness type Non Destructive Evaluation (NDE) is required. Parts may have to be disassembled and re-assembled for inspection, which can cause significant downtime for aircraft and contributes to a major part of the inspection effort. Increased downtime also accounts for the productivity costs. Disassembly and reassembly of structural components can also initiate damage and stress concentration zones at the structural joints. The damages in the structural plate can be one-sided damage or though-thickness crack, or delamination or fiber breakage (last two for composite specimen). These damages may or may not be accessible on a suitable surface for inspection with NDE equipment. These damages should be detected and localized at an early stage of development in order to prevent catastrophic failure of the structure. Identification of these damages at the incipient stage helps in replacement/maintenance of smaller component parts compared to replacement/maintenance of the structural component as a whole in the later stage. This prevents the propagation of the damage to nearby structural components and helps to increase the useful life of adjacent structural components. Therefore, rapid inspection, cost effective techniques and integrated SHM systems are needed with efficient use of accurate modeling and design of various parameters to overcome the shortcomings of the current inspection paradigm.

For diagnostics of damage one may use either vibration based methods or wave propagation methods. The sensitivity of vibration/modal analysis-based methods to detect small damage severities is comparatively less when compared to wave propagation based methods. The wave propagation method has been demonstrated to be effective in detecting small cracks, fatigue damage, corrosion in metallic structures and small size of debonding, delamination and matrix cracking in composites. Due to the presence of damage, there will be scattering and mode conversion of ultrasonic Lamb waves. These types of waves are elastic waves which can travel relatively long distances and through the entire thickness of the material with little amplitude loss and carry defect related features is considered for damage detection in the present method. One of the advantages of Lamb waves is that a relatively large structure can be investigated using a small number of transducers integrated to the structure as posed to physical scanning. Details of the methods to launch Lamb waves and type of wave modes in plate can be found in standard text [3]. Wave propagation in inhomogeneous and anisotropic media and application of wave based finite element method for efficient damage detection for structural health monitoring can be found in standard text [4]. The book also addresses new methods for the solution of wavenumbers for propagation in composites and inhomogeneous waveguides and the use of the spectral element for active vibration control. Diagnosis of damage characteristics based on measure of scattered energy using spectral power flow has been studied earlier by the author [5]. In that study, the frequency domain properties of the spectral power was shown to be quite informative. Effect of wave scattering due to delamination and strip inclusion on the frequency domain spectral power was discussed in that study. Mechanistic treatment of how the Lamb wave modes interact with delaminations and cracks in composite beams has been given in ref. [6]. At minute interfaces between heterogeneous stiffness and mass systems, considerable change in the near field effects may occur due to scattering of waves. One crucial outcome of such phenomena is the dynamic stress intensity at the delaminations tips. This may enhance the formation of delamination in the locations of severe stress discontinuities. This issue has been addressed by authors in ref. [7]. A spectral finite element model for analysis of axial-flexure-shear coupled wave propagation in laminated composite beams has been discussed in ref. [8]. A method of solving wavenumber using Polynomial Eigenvalue Problem (PEP) has been demonstrated in ref. [9].

The capabilities of small, inexpensive, non-obtrusive devices like embedded Piezoelectric Wafer Active Sensors (PWAS) to perform in-situ non-destructive evaluation using Lamb waves were initially demonstrated by Giurgiutiu [10]. There is a significant advantage of a sensor-actuator network which physically occupies less area on a structure but still monitors large area of the structure. Identification, parameterization and localization of various different damages using various sensor-actuator networks have been discussed in refs. [11-19]. Review of currently used NDT/NDE inspection methods and examples showing the detection of internal damage in multi-layered composite structures using Lamb wave technique can be found in [20] and references within. Application of Lamb wave techniques for detecting delamination, 
transverse ply cracks and through thickness holes in a quasi-isotropic graphite epoxy test specimen using piezo sensoractuator network has been reported by Kesseler et al. [21]. In this paper the authors have also focused on the optimal sensor-actuator configurations for illuminating the position of the damage.

For monitoring of very large structural component a significant number of actuator/sensor transducers are required. Irrespective of the price of the transducer that is used it is unrealistic to have a structure bonded with thousands of transducers. By using non-contact instruments for measuring the structural response the issues regarding liquid couplant, dry couplant, and sensitivity variation due to impedance mismatch between the couplant and the structure can be avoided. Among various different laser-based techniques for vibration measurement, such as holography, speckle pattern interferometry (ESPI), shearography, and laser Doppler vibrometery, the last one is the most widely investigated and used techniques at present. This is mainly because of its applicability in a wide range of testing situations with a long stand-off distance. Some of the early studies can be found in [22] and references therein. Application of 3-Dimensional LDV based measurements for quantitative NDE and SHM is an emerging area of research. By combining appropriate mathematical model with experimental data from LDV it is possible to locate and possibly quantify micro to millimeter scale defects. One common approach of collecting LDV data is to excite a simple fixed point using a conventional wired transducer and scan guided wave responses using a laser vibrometer. Sohn et al. [23] has used a completely non-contact system to excite and measure elastic waves in flat plate composite test specimen. In that study the author introduces the concept of standing wave filter and a Laplacian image filter to illuminate the zone of the damage with respect to its background wave field visualization. Detection of damages using LDV in various structures like plate specimen, composite structures by analyzing the change in signal pattern and various signal processing techniques has been discussed in ref. [24-29] and several other authors over last two decades. In the present paper the authors focus on computation of wavenumber for a third order plate theory using PEP and also focused on a method to diagnosis the structure without using baseline data.

\section{MATHEMATICAL FORMULATION}

In order to construct a theoretical framework to analyze the wave energy modes due to damage, we consider the combined effect of in-plane, out of plane and mode conversion. Understanding of Lamb wave mode conversion due to damage and interaction among these modes before and after scattering due to damage are not well reported in published literature. Investigation of the properties of the components of kinetic energy, strain energy and power flow for healthy and damaged condition is expected to provide new information which can further lead to new methodology of scanning and detection of damage by such scanning method and associated computations. Assuming a cubical displacement field

$$
\begin{aligned}
& u(x, y, z, t)=u_{0}(x, y, t)+z \phi_{x}(x, y, t)+z^{2} \psi_{x}(x, y, t)+z^{3} \xi_{x}(x, y, t) \\
& v(x, y, z, t)=v_{0}(x, y, t)+z \phi_{y}(x, y, t)+z^{2} \psi_{y}(x, y, t)+z^{3} \xi_{y}(x, y, t) \\
& w(x, y, z, t)=w_{0}(x, y, t)
\end{aligned}
$$

where $u_{0}, v_{0}$, and $w_{0}$ denote the displacements of a point ( $\left.\mathrm{x}, \mathrm{y}\right)$ on the midplane and $\phi_{x}$ and $\phi_{y}$ are the rotation of the normals to the midplane about the $\mathrm{y}$ and $\mathrm{x}$ axes, respectively. The functions $\psi_{x}, \psi_{y}, \xi_{x}$, and $\xi_{y}$ will be determined using the conditions transverse shear stress $\left.\tau_{x z}\right|_{\frac{h}{2}, \frac{-h}{2}}=0,\left.\tau_{y z}\right|_{\frac{h}{2}, \frac{-h}{2}}=0$ vanish at the boundaries. The displacement field in the equations (1)-(3) becomes, 


$$
\begin{aligned}
& u(x, y, z, t)=u_{0}(x, y, t)+z \phi_{x}(x, y, t)-\frac{4}{3 h^{2}} z^{3}\left(\phi_{x}+\frac{\partial w_{o}}{\partial x}\right) \\
& v(x, y, z, t)=v_{0}(x, y, t)+z \phi_{y}(x, y, t)-\frac{4}{3 h^{2}} z^{3}\left(\phi_{y}+\frac{\partial w_{o}}{\partial y}\right) \\
& w(x, y, z, t)=w_{0}(x, y, t)
\end{aligned}
$$

Following the conventional notation [31], constitutive model for orthotropic laminated composite plate can be expressed as

$$
\left\{\begin{array}{c}
\sigma_{x x} \\
\sigma_{y y} \\
\sigma_{z z} \\
\tau_{y z} \\
\tau_{x z} \\
\tau_{x y}
\end{array}\right\}=\left[\begin{array}{cccccc}
\bar{C}_{11} & \bar{C}_{12} & \bar{C}_{13} & 0 & 0 & \bar{C}_{16} \\
\bar{C}_{21} & \bar{C}_{22} & \bar{C}_{23} & 0 & 0 & \bar{C}_{26} \\
\bar{C}_{13} & \bar{C}_{23} & \bar{C}_{33} & 0 & 0 & \bar{C}_{36} \\
0 & 0 & 0 & \bar{C}_{44} & \bar{C}_{45} & 0 \\
0 & 0 & 0 & \bar{C}_{45} & \bar{C}_{55} & 0 \\
\bar{C}_{16} & \bar{C}_{26} & \bar{C}_{36} & 0 & 0 & \bar{C}_{66}
\end{array}\right]\left\{\begin{array}{c}
\varepsilon_{x x} \\
\varepsilon_{y y} \\
\varepsilon_{z z} \\
\gamma_{y z} \\
\gamma_{x z} \\
\gamma_{x y}
\end{array}\right\}
$$

Using Hamilton's principle governing wave equation can be obtained as

$$
\begin{aligned}
& \delta u_{0}:-A_{11} u_{0},{ }_{x x}-2 A_{16} u_{0},_{x y}-A_{66} u_{0},{ }_{y y}-A_{16} v_{0},_{x x}-\left[A_{12}+A_{66}\right] v_{0},{ }_{x y}-A_{26} v_{0},{ }_{y y}+\left[-B_{11}+c_{1} E_{11}\right] \phi_{x},{ }_{x x}+2 c_{1} E_{16} \phi_{x},{ }_{x y} \\
& +\left[-B_{66}+c_{1} E_{66}\right] \phi_{x},_{y y}+\left[B_{16}+c_{1} E_{16}\right] \phi_{y},_{x x}+\left[-B_{12}-B_{66}+c_{1} E_{12}+c_{1} E_{66}\right] \phi_{y},_{x y}+\left[-B_{26}+c_{1} E_{26}\right] \phi_{y},_{y y} \\
& +c_{1} E_{11} w_{0}, x x x+3 c_{1} E_{16} w_{0},{ }_{x x y}+\left[c_{1} E_{12}+2 c_{1} E_{66}\right] w_{0},{ }_{x y y}+c_{1} E_{26} w_{0},{ }_{y y y}+I_{0} \ddot{u}_{0}+\left(I_{1}-c_{1} I_{3}\right) \ddot{\phi}_{x}-c_{1} I_{3} \ddot{w}_{0},_{x}=0 \\
& \delta v_{0}:-A_{16} u_{0},_{x x}-\left[A_{12}+A_{66}\right] u_{0},_{x y}-A_{26} u_{0},_{y y}-A_{66} v_{0},_{x x}-2 A_{26} v_{0},_{x y}-A_{22} v_{0},_{y y}+\left[-B_{16}+c_{1} E_{16}\right] \phi_{x},_{x x} \\
& +\left[-B_{11}-B_{66}+c_{1} E_{12}+c_{1} E_{66}\right] \phi_{x},_{x y}+\left[B_{26}+c_{1} E_{26}\right] \phi_{x},{ }_{y y}+\left[-B_{66}+c_{1} E_{66}\right] \phi_{y},_{x x}+2 c_{1} E_{26} \phi_{y}, x_{x y} \\
& +\left[-B_{22}+c_{1} E_{22}\right] \phi_{y},{ }_{y y}+c_{1} E_{16} w_{0},{ }_{x x x}+\left[c_{1} E_{12}+2 c_{1} E_{66}\right] w_{0},{ }_{x x y}+\left[c_{1} E_{26}+2 c_{1} E_{26}\right] w_{0}, x y y+c_{1} E_{22} w_{0},{ }_{y y y} \\
& +I_{0} \ddot{v}_{0}+\left(I_{1}-c_{1} I_{3}\right) \ddot{\phi}_{y}-c_{1} I_{3} \ddot{w}_{0},{ }_{y}=0
\end{aligned}
$$




$$
\begin{aligned}
& \delta w_{0}:-c_{1} E_{11} u_{0},_{x x x}-c_{1}\left[E_{12}+2 E_{22}\right] u_{0},_{x y y}-3 c_{1} E_{16} u_{0},_{x x y}-c_{1} E_{26} u_{0},{ }_{y y y}-c_{1} E_{16} v_{0},{ }_{x x x}-3 c_{1} E_{26} v_{0},_{x y y} \\
& -c_{1}\left[E_{12}+2 E_{66}\right] v_{0},{ }_{x x y}-c_{1} E_{22} v_{0},{ }_{y y y}+c_{1}^{2} H_{11} w_{0},_{x x x x}+4 c_{1}^{2} H_{16} w_{0},{ }_{x x x y}+c_{1}^{2}\left[2 H_{12}+4 H_{66}\right] w_{0},{ }_{x x y y} \\
& +4 c_{1}^{2} H_{26} w_{0},_{x y y y}+c_{1}^{2} H_{22} w_{0},{ }_{y y y y}-c_{1}\left[F_{11}-c_{1} H_{11}\right] \phi_{x},_{x x x}+c_{1}\left[-3 F_{16}+3 c_{1} H_{16}\right] \phi_{x},_{x x y}+c_{1}\left[c_{1} H_{12}\right. \\
& \left.+2 c_{1} H_{66}-F_{12}-2 F_{66}\right] \phi_{x}, x y y+c_{1}\left[-F_{26}+c_{1} H_{26}\right] \phi_{x},{ }_{y y y}+c_{1}\left[-F_{16}+c_{1} H_{16}\right] \phi_{y},_{x x x} \\
& +3 c_{1}\left[-F_{26}+c_{1} H_{26}\right] \phi_{y},_{x y y}+c_{1}\left[-2 F_{66}+-F_{12}+c_{1} H_{12}+2 c_{1} H_{66}\right] \phi_{y}, x x y+c_{1}\left[-F_{22}+c_{1} H_{22}\right] \phi_{y},{ }_{y y y} \\
& +\left[-c_{2}^{2} F_{45}-A_{55}+2 c_{2} D_{55}-c_{2} F_{55}\right] w_{0},_{x x}+\left[-A_{44}+2 c_{2} D_{44}-c_{2}^{2} F_{44}\right] w_{0},_{y y}+\left[-2 A_{45}+4 c_{2} D_{45}\right. \\
& \left.-2 c_{2}^{2} F_{45}\right] w_{0},{ }_{x y}+\left[-A_{44}+2 c_{2} D_{44}-c_{2}^{2} F_{44}\right] \phi_{y}, y+\left[-A_{45}+2 c_{2} D_{45}-c_{2}^{2} F_{45}\right] \phi_{x}, y_{y} \\
& +\left[-A_{55}+2 c_{2} D_{55}-c_{2}^{2} F_{55}\right] \phi_{x},{ }_{x}+\left[-A_{45}+2 c_{2} D_{45}-c_{2}{ }^{2} F_{45}\right] \phi_{y},{ }_{x}+c_{1} I_{3} \ddot{u}_{0},{ }_{x}+c_{1} I_{3} \ddot{v}_{0}, y_{y} \\
& +c_{1}\left(I_{4}-c_{1} I_{6}\right) \ddot{\phi}_{x},{ }_{x}-c_{1}^{2} I_{6} \ddot{w}_{0},{ }_{x x}+c_{1}^{2} I_{6} \ddot{\phi}_{y},{ }_{y}-c_{1}^{2} I_{6} \ddot{w}_{0},{ }_{y y}+c_{1} I_{4} \ddot{\phi}_{x},{ }_{y}+I_{0} \ddot{w}_{0}=0
\end{aligned}
$$

$\delta \phi_{x}:\left[-B_{11}+c_{1} E_{11}\right] u_{0},_{x x}+\left[-B_{16}-A_{16}+2 c_{1} E_{16}\right] u_{0},_{x y}+\left[-A_{66}+c_{1} E_{66}\right] u_{0},{ }_{y y}+\left[-B_{16}+c_{1} E_{16}\right] v_{0},_{x x}$

$$
\begin{aligned}
& +\left[-B_{12}-A_{66}+c_{1} E_{12}++c_{1} E_{66}\right] v_{0},_{x y}+\left[-A_{26}+c_{1} E_{26}\right] v_{0},_{y y}+c_{1}\left[F_{11}-c_{1} H_{11}\right] w_{0}, x x x \\
& +c_{1}\left[2 F_{16}+c_{1} E_{16}-2 c_{1} H_{16}\right] w_{0},_{x x y}+c_{1}\left[F_{12}+2 c_{1} E_{66}-c_{1} H_{12}-2 c_{1} H_{66}\right] w_{0},_{x y y}+c_{1}\left[E_{26}-c_{1} H_{26}\right] w_{0},_{y y y} \\
& +\left[-D_{11}+2 c_{1} F_{11}-c_{1}^{2} H_{11}\right] \phi_{x},_{x x}+\left[-B_{26}+c_{1} E_{26}+c_{1} F_{26}-c_{1}^{2} H_{26}\right] \phi_{y},_{y y}+\left[-D_{16}+2 c_{1} F_{16}-c_{1}^{2} H_{16}\right] \phi_{y},_{x x} \\
& +\left[-B_{66}+c_{1} E_{66}+c_{1} F_{66}-c_{1}^{2} H_{66}\right] \phi_{x},{ }_{y y}+\left[-D_{12}+2 c_{1} F_{12}-c_{1}^{2} H_{66}-c_{1}^{2} H_{12}+c_{1} E_{66}+c_{1} F_{66}-B_{66}\right] \phi_{y},_{x y} \\
& +\left[-D_{16}+3 c_{1} F_{16}-B_{16}+c_{1} E_{16}-2 c_{1}^{2} H_{16}\right] \phi_{x},_{x y}+\left[-2 c_{2} D_{45}+c_{2}^{2} F_{45}+A_{45}\right] w_{0},_{y} \\
& +\left[-2 c_{2} D_{55}+c_{2}^{2} F_{55}+A_{55}\right] w_{0},_{x}+\left[-2 c_{2} D_{55}+c_{2}^{2} F_{55}+A_{55}\right] \phi_{x}+\left[-2 c_{2} D_{45}+c_{2}{ }^{2} F_{45}+A_{45}\right] \phi_{y} \\
& +\left(I_{1}-c_{1} I_{3}\right) \ddot{u}_{0}+\left(I_{2}-2 c_{1} I_{4}+c_{1} I_{6}\right) \ddot{\phi}_{x}+c_{1}\left[I_{6}-I_{4}\right] \ddot{w}_{0},_{x}=0
\end{aligned}
$$

$\delta \phi_{y}:\left[-A_{16}+c_{1} E_{16}\right] u_{0},{ }_{x x}+\left[-B_{12}-A_{66}+c_{1} E_{12}+c_{1} E_{66}\right] u_{0},{ }_{x y}+\left[-B_{26}+c_{1} E_{26}\right] u_{0},{ }_{y y}+\left[-A_{66}+c_{1} E_{66}\right] v_{0},{ }_{x x}$ $+\left[-B_{26}-A_{26}+2 c_{1} E_{26}\right] v_{0},{ }_{x y}+\left[-A_{22}+c_{1} E_{22}\right] v_{0},{ }_{y y}+c_{1}\left[E_{16}-c_{1} H_{16}\right] w_{0},{ }_{x x x}+c_{1}\left[F_{12}+2 E_{66}-c_{1} H_{12}\right.$ $\left.-2 c_{1} H_{66}\right] w_{0},{ }_{x x y}+c_{1}\left[2 F_{26}-3 c_{1} H_{26}+c_{1} E_{26}\right] w_{0},{ }_{x y y}+c_{1}\left[F_{22}-c_{1} H_{22}\right] w_{0},{ }_{y y y}+\left[-B_{16}+c_{1} E_{16}+c_{1} F_{16}\right.$ $\left.-c_{1}^{2} H_{16}\right] \phi_{x},_{x x}+\left[-D_{22}+2 c_{1} F_{22}-c_{1}^{2} H_{22}\right] \phi_{y},{ }_{y y}\left[-D_{26}+2 c_{1} F_{26}-c_{1}^{2} H_{26}\right] \phi_{x}, y y+\left[-B_{66}+c_{1} E_{66}\right.$ $\left.+c_{1} F_{66}-c_{1}^{2} H_{66}\right] \phi_{y},{ }_{x x}+\left[-D_{26}-B_{26}+3 c_{1} F_{26}+c_{1} E_{26}-2 c_{1}^{2} H_{26}\right] \phi_{y},{ }_{x y}+\left[-2 c_{2} D_{44}+c_{2}^{2} F_{44}+A_{44}\right] w_{0},{ }_{y}$ $\left[-D_{12}-B_{66}+2 c_{1} F_{12}+c_{1} E_{66}+c_{1} F_{66}-c_{1}^{2} H_{12}-c_{1}^{2} H_{66}\right] \phi_{x},_{x y}+\left[-2 c_{2} D_{44}+c_{2}^{2} F_{44}+A_{44}\right] \phi_{y}$ $+\left[-2 c_{2} D_{45}+c_{2}{ }^{2} F_{45}+A_{45}\right] w_{0}{ }_{x}+\left[-2 c_{2} D_{45}+c_{2}{ }^{2} F_{45}+A_{45}\right] \phi_{x}+\left(I_{1}-c_{1} I_{3}\right) \ddot{v}_{0}+\left(I_{2}-2 c_{1} I_{4}+c_{1} I_{6}\right) \ddot{\phi}_{y}$ $+c_{1}\left[I_{6}-I_{4}\right] \ddot{w}_{0},{ }_{y}=0$ 
where $c_{1}=\frac{4}{3 h^{2}}, c_{2}=\frac{4}{h^{2}}$ and $\langle\rangle,._{x}$ and $\langle\rangle,._{y}$ are derivatives of the field variables with respect to $\mathrm{x}$ and $\mathrm{y}$, respectively. The stiffness coefficients which are the functions of individual ply properties, ply orientations etc. and are integrated over the beam cross-section, can be expressed as

$$
\left[A_{i j}, B_{i j}, D_{i j}, E_{i j}, F_{i j}, H_{i j}\right]=\sum_{k=1}^{N} \int_{Z_{k}}^{Z_{k+1}} \frac{(k)}{C_{i j}}\left[1, z, z^{2}, z^{3}, z^{4}, z^{6}\right] d z
$$

The coefficients associated with the inertial terms can be expressed as

$$
I_{i}=\int_{\frac{-h}{2}}^{\frac{h}{2}} \rho_{0}(z)^{i} d z \quad(i=1,2, \ldots, 6)
$$

Using Discrete Fourier Transformation (DFT) for the temporal field, the spectral solution for primary displacement field variables can be expressed as

$$
u_{0}(x, y, t)=\sum_{n=1}^{N} \hat{u}_{0}\left(x, w_{n}\right) e^{i w_{n} t}=\sum_{n=1}^{N}\left(\tilde{u}_{0_{j}} e^{-i\left(k_{x_{j}} x+k_{y_{j}} y\right)}\right) e^{i w_{n} t}
$$

Substituting the assumed form in the equation (15) and by imposing the condition $\mathrm{k}_{\mathrm{y}}=0$, a Polynomial Eigenvalue Problem (PEP) is posed as in equation (16).

$$
\left\{A_{0}+A_{1} k_{x_{j}}+A_{1} k_{x_{j}}^{2}+A_{1} k_{x_{j}}^{3}+A_{1} k_{x_{j}}^{4}\left\{\begin{array}{c}
\widetilde{u}_{0_{j}} \\
\widetilde{v}_{0_{j}} \\
\widetilde{w}_{0_{j}} \\
\widetilde{\phi}_{x_{j}} \\
\widetilde{\phi}_{y_{j}}
\end{array}\right\}=0\right.
$$

where $A_{0}, A_{1}, A_{2}, A_{3}$, and $A_{4}$ is given in expression (17)-(21) 


\begin{tabular}{|c|c|c|c|c|}
\hline$-I_{0} \omega^{2}$ & 0 & 0 & $-\left(I_{1}-c_{1} I_{3}\right) \omega^{2}$ & 0 \\
\hline 0 & $-I_{0} \omega^{2}$ & 0 & 0 & $-\left(I_{1}-c_{1} I_{3}\right) \omega^{2}$ \\
\hline 0 & 0 & $-I_{0} \omega^{2}$ & 0 & 0 \\
\hline$-\left(I_{1}-c_{1} I_{3}\right) \omega^{2}$ & 0 & 0 & $\begin{array}{l}\left(-2 c_{2} D_{55}+c_{2}^{2} F_{55}+A_{55}\right) \\
-\left(I_{2}-2 c_{1} I_{4}+c_{1} I_{6}\right) \omega^{2}\end{array}$ & $-2 c_{2} D_{45}+c_{2}^{2} F_{55}+A_{55}$ \\
\hline 0 & $-\left(I_{1}-c_{1} I_{3}\right) \omega^{2}$ & 0 & $\left(A_{45}-2 c_{2} D_{45}+c_{2}^{2} F_{45}\right)$ & $\begin{array}{l}\left(A_{44}-2 c_{2} D_{44}+c_{2}^{2} F_{44}\right) \\
-\left(I_{2}-2 c_{1} I_{4}+c_{1} I_{6}\right) \omega^{2}\end{array}$ \\
\hline
\end{tabular}

\begin{tabular}{|c|c|c|c|c|}
\hline 0 & 0 & $-i c_{1} I_{3} \omega^{2}$ & 0 & 0 \\
\hline 0 & 0 & 0 & 0 & 0 \\
\hline$-i c_{1} I_{3} \omega^{2}$ & 0 & 0 & $\begin{array}{l}i\left(A_{55}-2 c_{2} D_{55}+c_{2}^{2} F_{55}\right) \\
+i\left(c_{1} I_{4}-c_{1}^{2} I_{6}\right) \omega^{2}\end{array}$ & $-i\left(-A_{45}+2 c_{2} D_{45}-c_{2}^{2} F_{45}\right)$ \\
\hline 0 & $-i\left[-B_{16}+c_{1} E_{16}\right]$ & $\begin{array}{c}-i\left(A_{55}-2 c_{2} D_{55}+c_{2}^{2} F_{55}\right) \\
-i c_{1}\left(I_{4}-I_{6}\right) \omega^{2}\end{array}$ & 0 & 0 \\
\hline 0 & 0 & $-i\left(A_{45}-2 c_{2} D_{45}+c_{2}^{2} F_{45}\right)$ & 0 & 0 \\
\hline
\end{tabular}

\begin{tabular}{|c|c|c|c|c|}
\hline$A_{11}$ & $A_{16}$ & 0 & $-\left[-B_{11}+c_{1} E_{11}\right]$ & $-\left[-B_{16}+c_{1} E_{16}\right]$ \\
\hline$A_{16}$ & $A_{66}$ & 0 & $-\left[-B_{16}+c_{1} E_{16}\right]$ & {$\left[-c_{1} E_{66}+B_{66}\right]$} \\
\hline 0 & 0 & $\begin{array}{c}\left(A_{55}-2 c_{2} D_{55}+c_{2} F_{55}\right) \\
-c^{2} I_{6} \omega^{2}\end{array}$ & 0 & 0 \\
\hline$-\left[-B_{11}+c_{1} E_{11}\right]$ & 0 & 0 & $-\left(-D_{1}+2 c_{1} F_{11}-c_{1}^{2} H_{11}\right)$ & $\left(-2 c_{1} F_{16}+c_{1}^{2} H_{16}+D_{16}\right)$ \\
\hline$-\left[-A_{16}+c_{1} E_{16}\right]$ & $-\left[-A_{66}+c_{1} E_{66}\right]$ & 0 & $\left(A_{44}-2 c_{2} D_{44}+c_{2}^{2} F_{44}\right)$ & $-\left(c_{1} E_{66}+c_{1} F_{66}-c_{2}^{2} H_{66}-B_{66}\right)$ \\
\hline
\end{tabular}

$A_{3}=\left[\begin{array}{ccccc}0 & 0 & i c_{1} E_{11} & 0 & 0 \\ 0 & 0 & i c_{1} E_{16} & 0 & 0 \\ -i c_{1} E_{11} & -i c_{1} E_{11} & 0 & i\left(-c_{1} F_{11}+c_{1}^{2} H_{11}\right) & i\left(-c_{1} F_{16}+c_{1}^{2} H_{16}\right) \\ 0 & 0 & i\left(c_{1} F_{11}-c_{1}^{2} H_{11}\right) & 0 & 0 \\ 0 & 0 & i\left(c_{1} E_{16}-c_{1}^{2} H_{16}\right) & 0 & 0\end{array}\right](20)$




$$
A_{4}=\left[\begin{array}{ccccc}
0 & 0 & 0 & 0 & 0 \\
0 & 0 & 0 & 0 & 0 \\
0 & 0 & i c_{1}^{2} H_{11} & 0 & 0 \\
0 & 0 & 0 & 0 & 0 \\
0 & 0 & 0 & 0 & 0
\end{array}\right]
$$

The wavenumber dispersion obtained after solving PEP as in equation (16) is given in figure 1.

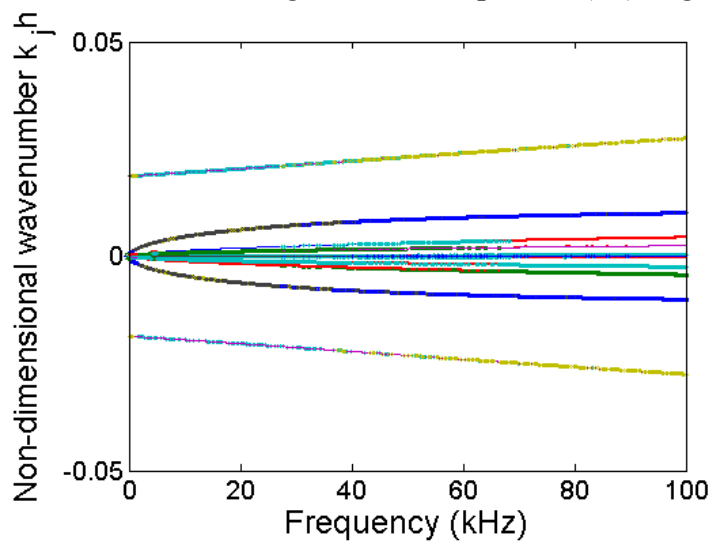

Figure 1: Non-dimensional wavenumber dispersion obtained for a $3^{\text {rd }}$ order plate theory.

\section{EXPERIMENTAL SETUP AND PROCEDURE}

The dimensions of flange and web of the T-joint test specimen are $260 \mathrm{~mm}$ x $125 \mathrm{~mm} \times 2 \mathrm{~mm}$ and $260 \mathrm{~mm} \times 75 \mathrm{~mm} \times 2$ $\mathrm{mm}$, respectively. The test specimen was excited using a Lead-Zirconate-Titanate (PZT) wafer active patch which was bonded on to the back face of the accessible flange (see figure 2(b)). Piezo actuator is bonded to the plate sample using a removable polymer bonding, so that they are firmly attached during experiments, but could be removed afterwards to recover them. The dimensions of PZT wafer patch are $15 \mathrm{~mm}$ diameter and $2 \mathrm{~mm}$ thickness. Other forms and size of transducer patches can be used with information regarding their mechanisms of generating specific modes of Lamb waves. Actuation was carried out using a PXI control card. A Labview-Matlab interface code was created for this purpose, which launches a programmed waveform (e.g., a tone-burst signal with known frequency content and amplitude). Signal acquisition was performed using a Polytec PSV-400-M 3D Laser Doppler Vibrometer. Experimental setup for scanning T-joint test specimen using 3D LDV is shown in figure 2(a). To remove the unnecessary noises in the signal, a band-pass filter was used. Actuation using piezo actuator and sensing using LDV is synchronized by using a trigger setup. Due to good dispersion characteristics and structural flaw sensitivity, five cycle windowed sine-burst signal is used for damage detection. A Hanning time window is used for this which concentrates maximum amount of energy at the desired driving frequency.

By using non-contact laser vibrometery it is possible to have seamless number of scanning points. The grid size for scanning is controlled using the LDV software. The plate structure is preliminarily scanned using a coarse mesh to obtain the suspected region of damage. Near the suspected region of damage, scanning with a fine mesh is done. The grid spacing should be at least one-tenth of wavelength $(\lambda)$. $\lambda$ is computed based on the frequency of excitation and 
corresponding group velocity of Lamb wave. The results obtained using LDV are much more accurate in a sense that complicated calculation using analytical model for bonded PZT wafer as sensors and associated calibration can be avoided. Velocity/displacement components in X, Y, and Z direction are obtained from 3D LDV. The Z component gives the out-of-plane component while the $\mathrm{X}$ and $\mathrm{Y}$ component gives the in-plane components. The raw measurements from the three scanning heads are combined with the beam angle in the data processing computer in order to resolve the velocity data onto an orthogonal $(\mathrm{X}, \mathrm{Y}, \mathrm{Z}) 3 \mathrm{D}$ coordinate system. The Cartesian coordinate system with respect to LDV laser heads for the T-joint test specimen is shown in figure 3.

\begin{tabular}{|l|l|}
\hline \multicolumn{2}{|c|}{ Test Settings } \\
\hline Excitation frequency & $100 \mathrm{kHz}$ \\
\hline Filter & Bandpass filter $(97.5 \mathrm{kHz}-102.5 \mathrm{kHz})$ \\
\hline Sampling Frequency & $2.56 \mathrm{MHz}$ \\
\hline Number of samples & 1024 \\
\hline Sample Time & $400 \mu \mathrm{s}$ \\
\hline Pre-trigger & $10 \%$ Sample time \\
\hline Total scan points & 30761 \\
\hline Range of decoder & VD-07 50mm/s/V \\
\hline Excitation Voltage & $3 \mathrm{~V}(\mathrm{Gain} 3.5)$ \\
\hline Time delay between each trigger $(\mathrm{dt})$ & $5 \mathrm{sec}$ \\
\hline
\end{tabular}

Table 1. Acquisition settings details for T-joint test specimen using 3D LDV.

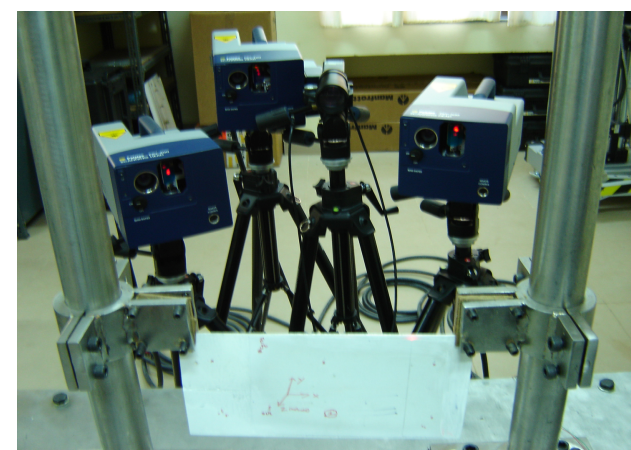

(a)

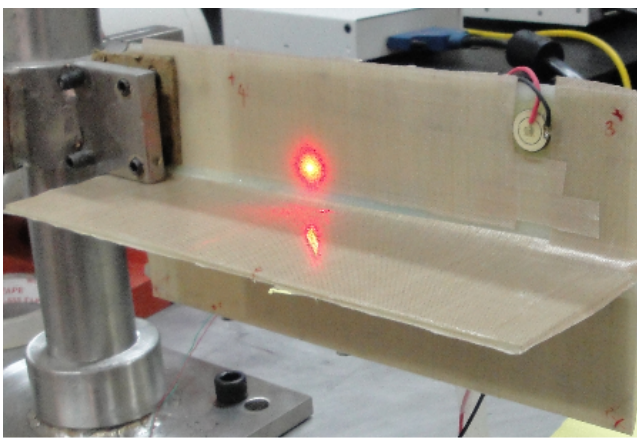

(b)

Figure 2: (a) Experimental setup showing T-joint mounted on the vertical stands in front of scanning LDV. (b) Enlarged view of T-joint test specimen with delamination and bonded piezo actuator for excitation of the structure.

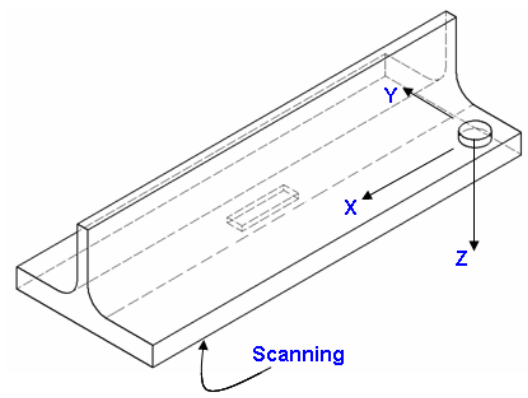

Figure 3: The Cartesian coordinate system with respect to LDV laser heads for the T-joint test specimen. 


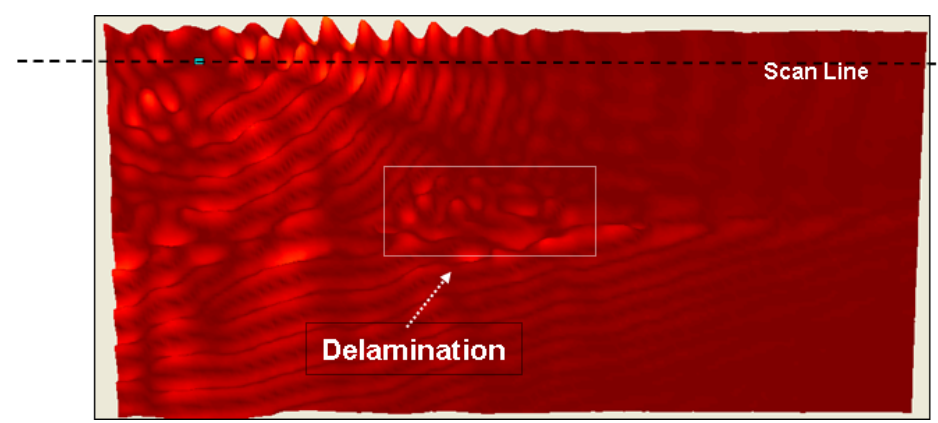

Figure 4: The figure depicts the fringe pattern obtained in a T-joint test specimen with delamination.

\section{RESULTS AND DISCUSSIONS}

The Power flow is defined as the product of force vector and complex conjugate of velocity vector at a material point (see ref [5]).

$$
\begin{aligned}
& \hat{P}=\frac{1}{2} \hat{f}^{T}\left[i w_{n} \hat{u}\right]^{*} \\
& \hat{P}=-\frac{1}{2} i w_{n}\left[\left(A_{11} \hat{u},_{x}\right) \hat{u}^{*}+\left(A_{22} \hat{v},_{x}\right) \hat{v}^{*}+\left(A_{55} \hat{w},{ }_{x}\right) \hat{w}^{*}\right]
\end{aligned}
$$

where $i=\sqrt{-1}, w_{n}$ is the frequency at the $\mathrm{n}^{\text {th }}$ sampling point. In the current experiment a scan line over a healthy zone has been defined as shown in figure 4 . The velocity components obtained from non-contact 3D LDV is post-processed to obtain the power flow along the scan line (see figure 5). In this analysis the effect of S0 mode, A0 mode and mode conversion has been taken into account. The location of any change in material/geometric properties can be traced by computing power flow along the scan line. It can be seen from the figure that power flow along the scan line is invariant. The abnormal variation in the graph is due to the low Signal to Noise Ratio (SNR). The power flow along the scan line is approximately zero along the healthy scan line. The proposed technique eliminates the necessity of baseline signal for damage identification and localization.

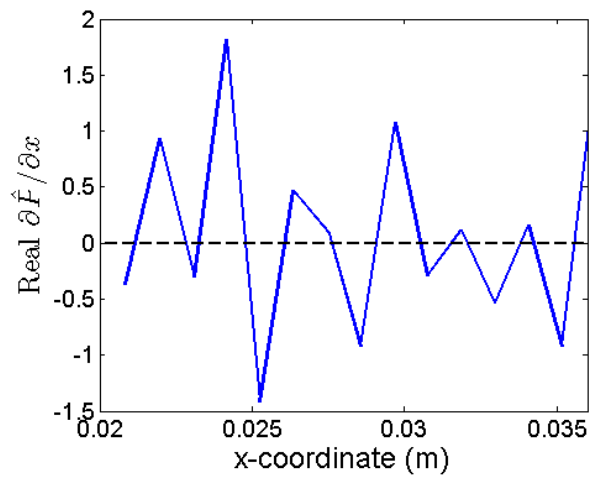

Figure 5: The figure shows the invariance of the power flow obtained at diiferent scan points along the scan line. 


\section{CONCLUSION}

A mathematical model for axial-flexure-shear coupled wave propagation in a laminated composite plate using $3^{\text {rd }}$ order plate theory with arbitrary ply-stacking sequence has been developed in the present paper and it has been further employed to develop an LDV based quantitative technique. In this analysis the effect of S0 mode, A0 mode and mode conversion has been taken into account. The location of any change in material/geometric properties can be traced by computing power flow along the scan line. The proposed technique eliminates the necessity of baseline signal for damage identification and localization. This method of damage interrogation can be further extended to curved structures, complex geometry structures. Important observations which are brought out by authors in this paper will open up future potentials toward the application of non-contact laser vibrometery for guided wave based structural health

monitoring. This approach is well amenable within the anticipated framework of Integrated Vehicle Health Monitoring (IVHM).

\section{Acknowledgements}

The authors gratefully acknowledge financial support under the PARC 3, NPMASS Program, Government of India.

\section{REFERENCES}

[1] Brand, C. and Boller, C., "Identification of life cycle cost reduction potentials of future self diagnostic structures", RTO Applied Vehicle Technology Panel (1999).

[2] LaRiviere, S. G., and Thompson, J., "Development of reliable NDI procedures for airframe inspection" NATO RTO Meeting Proc. 10: Airframe Inspection Reliability under Field/Depot Conditions (1998).

[3] Rose, J. L., "Ultrasonic waves in solid media", Cambridge, U.K, Cambridge University Press (1999).

[4] Gopalakrishnan, S., Chakraborty, A. and Roy Mahapatra, D., "Spectral Finite Element Method: Wave propagation, diagnostics and control in anisotropic and inhomogeneous structures", Springer, Berlin (2007).

[5] Roy Mahapatra, D. and Gopalakrishnan, S., "Spectral finite element analysis of coupled wave propagation in composite beams with multiple delaminations and strip inclusions", International Journal of Solids and Structures, 41, 1173-1208 (2004).

[6] Kumar, D. S., Roy Mahapatra, D. and Gopalakrishnan, S., "A spectral finite element for wave propagation and structural diagnostic analysis of composite beam with transverse crack", Finite Element in Analysis and Design, 40, 1729-1751 (2004).

[7] Chakraborty, A., Roy Mahapatra, D. and Gopalakrishnan, S., "Finite element analysis of free vibration and wave propagation in asymmetric composite beams with structural discontinuities", Composite Structures, 10, 10461058 (2001).

[8] Roy Mahapatra, D. and Gopalakrishnan, S., "A spectral finite element model for analysis of axial-flexure-shear coupled wave propagation in laminated composite beams", Composite Structures, 59, 67-88 (2003).

[9] Chakraborty, A. and Gopalakrishnan, S., "A spectrally formulated plate element for wave propagation analysis in anisotropic material", Computer Methods in Applied Mechanics and Engineering, 194, 4425-4446 (2005).

[10] Giurgiutiu, V., "Embedded NDE with piezoelectric wafer-active sensors in aerospace applications", Journal of Materials (JOM), online special issue on Nondestructive Evaluation (2003).

[11] Rathod, V. T., Roy Mahapatra, D. and Gopalakrishnan, S., "A circular array of piezoelectric sensors for lamb wave based structural health monitoring", IISc Centenary International Conference and Exhibition on Aerospace Engineering, ICEAE, Bangalore, India (2009). 
[12] Rathod, V.T., Roy Mahapatra, D. and Gopalakrishnan, S., "Lamb wave based identification and parameter estimation of corrosion in metallic plate structure using a circular PWAS array", Health Monitoring of Structural and Biological Systems, Proceedings of SPIE Vol. 7295, 72951C (2009).

[13] Giridhara, G., Rathod, V.T., Naik, S., Roy Mahapatra, D. and Gopalakrishnan, S., “ Rapid localization of damage using a circular sensor array and lamb wave based triangulation", Mechanical Systems and Signal Processing, 24, 2929-2946 (2010).

[14] Kolappan Geetha, G., Rathod, V. T., Chakraborty, N., Roy Mahapatra, D. and Gopalakrishnan, S., "Rapid localization and ultrasonic imaging of multiple damages in a structural panel with piezoelectric sensor-actuator network", Proceeding of IWSHM, Vol. 1, 249-256 (2011).

[15] Rathod, V.T. and Roy Mahapatra, D., "Lamb wave based monitoring of plate-stiffener debonding using a circular array of piezoelectric sensors", International Journal On Smart Sensing and Intelligent Systems, 3(1), 27-44 (2010).

[16] Kolappan Geetha, G., Rathod, V. T., Roy Mahapatra, D. and Gopalakrishnan, S., "Lamb wave based detection of damage in a stiffener bonded to the plate", Health Monitoring of Structural and Biological Systems, Proceedings of SPIE Vol. 7984, 79842X (2011).

[17] Kim, D. and Philen, M., "Guided wave beam steering using MFC phased arrays for structural health monitoring: analysis and experiment", Journal of Intelligent Material Systems and Structures, 21, 1011-1024 (2010).

[18] Purekar, A. S. and Pines, D. J., "Damage detection in thin composite laminates using piezoelectric phased sensor arrays and guided lamb wave interrogation", Journal of Intelligent Material Systems and Structures, 21, 995-1010 (2010).

[19] Rathod, V. T., Chakraborty, N. and Roy Mahapatra, D., "Linear phased array of piezoelectric transducers for delamination monitoring in a composite laminate using lamb waves", Smart Sensor Phenomena, Technology, Networks, and Systems, Proceedings of SPIE Vol. 7982, 798217 (2011).

[20] Diamanti, K. and Soutis, C., "Structural health monitoring techniques for aircraft composite structures", Progress in Aerospace Sciences, 46, 342-352 (2010).

[21] Kesseler, S.S., Spearing, S. M. and Soutis, C., "Damage detection in composite materials using lamb wave methods", Smart Materials and Structures, 11, 269-278 (2002).

[22] Sriram, P., "Whole field optical methods for structural mechanics: digital speckle correlation and laser doppler velocimetry", PhD Thesis, Georgia Institute of Technology (1988).

[23] Sohn, H., Dutta, D., Yang, J. Y., Park, H. J, DeSimio, M., Olson, S., and Swenson, E., "Delamination detection in composites through wave field image processing", Composite Science and Technology, 71, 1250-1256 (2011).

[24] Sharma, V. K., "Laser doppler vibrometer for efficient structural health monitoring" PhD Thesis, Georgia Institute of Technology (2008).

[25] Ruzzene, M., "Frequency-wavenumber domain filtering for improved damage visualization", Smart Materials and Structures, 16, 2116-2129 (2007).

[26] Staszewski, W. J., Lee, B.C., Mallet, L. and Scarpa, F., "Structural health monitoring using scanning laser vibrometery: I. Lamb wave sensing", Smart Materials and Structures, 13, 251-260 (2004).

[27] Mallet, L., Lee, B.C, Staszewski, W. J. and Scarpa, F., "Structural health monitoring using scanning laser vibrometery: II. Lamb waves for damage detection", Smart Materials and Structures, 14, 1387-1395 (2005).

[28] Leong, W. H., Staszewski, W. J., Lee, B. C. and Scarpa, F., "Structural health monitoring using scanning laser vibrometery: III. Lamb waves for fatigue crack detection", Smart Materials and Structures, 13, 261-269 (2004).

[29] Staszewski, W. J, Lee, B. C and Traynor, R., "Fatigue crack detection in metallic structures with Lamb waves and 3D laser vibrometry", Measurement Science and Technology, 18, 727-739 (2007).

[30] "Polytech GmbH: Non-contact vibration measurement," available on Polytech's website at http:// http://www.polytec.com/us/news/infocus-magazine/ (2010).

[31] Reddy, J. N., "Mechanics of Laminated Composite Plates", CRC press (1997). 